\title{
ON STABILITY OF UNITARY MATRIX SPACES
}

\author{
JONATHAN ARAZY
}

\begin{abstract}
Stability (in the sense of Krivine-Maurey) of a symmetric sequence $E$ is equivalent to stability of the associated unitary matrix space $C_{E}$.
\end{abstract}

In what follows $E$ will denote a symmetric sequence space, i.e., a Banach space of sequences such that the standard unit vectors $\left\{e_{n}\right\}_{n=1}^{\infty}$ (defined by $e_{n}(j)=\delta_{n, j}$ ) form a normalized, 1-symmetric basis of $E$. $C_{E}$ will denote the associated unitary matrix space, i.e., the Banach space of all compact operators $x$ on $l_{2}$ so that $s(x) \in$ $E$, normed by $\|x\|_{C_{E}}=\|s(x)\|_{E}$. Here $s(x)=\left(s_{i}(x)\right)_{i=1}^{\infty}$ is the sequence of $s$-numbers of $x$, i.e., the eigenvalues of $\left(x^{*} x\right)^{1 / 2}$, arranged in a nonincreasing ordering, counting multiplicity.

In [1] we studied, among other topics, basic sequences in unitary matrix spaces. The main result concerning them is that every basic sequence in $C_{E}$ has a subsequence equivalent to a basic sequence in $l_{2} \oplus E$ (see [1, Theorem 2.4 and Corollary $2.8])$. This theorem and its variants proved to be a powerful tool in reducing the study of properties of $C_{E}$ which depend on the asymptotic behavior of sequences to that of the analogous properties of $E$.

In this paper we apply the methods from [1] to reduce the study of stability of $C_{E}$ to that of $E$.

DEFINITION [2]. A Banach space $X$ is said to be stable if, for every pair $\left\{x_{n}\right\}_{n=1}^{\infty}$, $\left\{y_{n}\right\}_{n=1}^{\infty}$ of bounded sequences in $X$ and for every pair of ultrafilters $\mathcal{U}, \mathcal{V}$ on the set $\mathbf{N}$ of positive integers, one has

$$
\lim _{m, \mathcal{V}}\left(\lim _{n, \mathcal{U}}\left\|x_{n}+y_{m}\right\|\right)=\lim _{n, \mathcal{U}}\left(\lim _{m, \mathcal{V}}\left\|x_{n}+y_{m}\right\|\right) .
$$

Our main result is the following:

THEOREM. Let $E$ be any symmetric sequence space. Then $E$ is stable if and only if $C_{E}$ is stable.

We remark that $E$ is isometric to a subspace of $C_{E}$ (consider the diagonal matrices in $C_{E}$ ); so stability of $C_{E}$ implies that of $E$. Thus we have to prove that stability of $E$ implies that of $C_{E}$.

First, recall the following:

Proposition 1 [2]. A Banach space $X$ is stable if..and only if for every pair $\left\{x_{n}\right\}_{n=1}^{\infty},\left\{y_{n}\right\}_{n=1}^{\infty}$ of bounded sequences in $X$,

$$
\inf _{n>m}\left\|x_{n}+y_{m}\right\| \leq \sup _{n<m}\left\|x_{n}+y_{m}\right\|
$$

Received by the editors November 17, 1981.

1980 Mathematics Subject Classification. Primary 47D15; Secondary 47B10, 46B99.

(c) 1983 American Mathematical Society 0002-9939/82/0000-0265/\$02.25 
Proposition 2 [3, ThEOREM 1]. Every stable Banach space is weakly sequentially complete.

Next, let $\left\{e_{i, j}\right\}_{i, j=1}^{\infty}$ denote the standard unit matrices (i.e., $\left.e_{i, j}(k, l)=\delta_{i, k} \cdot \delta_{j, l}\right)$, and let $S_{n}=\operatorname{span}\left\{e_{i, j} ; \max \{i, j\}=n\right\}$. The sequence $\left\{S_{n}\right\}_{n=1}^{\infty}$ is a monotone Schauder decomposition of $C_{E}$, called the shell decomposition.

COROLlARY 3. Let $E$ be a stable symmetric sequence space. Then $C_{E}$ does not contain a subspace isomorphic to $c_{0}$, and the shell decomposition of $C_{E}$ is boundedly complete.

Proof. By Proposition 2, $E$ is weakly sequentially complete, and so $c_{0} \nsubseteq E$. The rest follows from [1, Proposition 3.7].

The main tool needed for the proof of the Theorem is the following lemma (here $l_{2}$ is identified with $l_{2} \oplus l_{2}$ in a canonical way).

LEMMA 4. Assume that $c_{0} \nsubseteq E$, and let $\left\{x_{n}\right\}_{n=1}^{\infty}$ and $\left\{y_{n}\right\}_{n=1}^{\infty}$ be bounded sequences in $C_{E}$. Then for every $\epsilon>0$ there exist two sequences $\left\{x_{k}^{\prime}\right\}_{k=2}^{\infty},\left\{y_{k}^{\prime}\right\}_{k=2}^{\infty}$ in $C_{E}$, two unitary operators $u$ and $v$, and an increasing sequence $\left\{n_{k}\right\}_{k=2}^{\infty}$ of positive integers so that

(i) for some $\left\{x_{i, j}\right\}_{i, j=1}^{2}$ and $\left\{z_{j}\right\}_{k=2}^{\infty}$ in $C_{E}$ we have, for all $k \geq 2$,

$$
\begin{aligned}
u x_{k}^{\prime} v= & x_{1,1} \otimes e_{1,1}+x_{1,2} \otimes e_{1,2 k-1}+x_{2,1} \otimes e_{2 k-1,1} \\
& +x_{2,2} \otimes e_{2 k-1,2 k-1}+z_{k} \otimes e_{2 k, 2 k}
\end{aligned}
$$

(ii) for some $\left\{y_{i, j}\right\}_{i, j=1}^{2}$ and $\left\{w_{k}\right\}_{k=2}^{\infty}$ in $C_{E}$ we have, for all $k \geq 2$,

$$
\begin{gathered}
u y_{k}^{\prime} v=y_{1,1} \otimes e_{1,1}+y_{1,2} \otimes e_{1,2 k-1}+y_{2,1} \otimes e_{2 k-1,1} \\
+y_{2,2} \otimes e_{2 k-1,2 k-1}+w_{k} \otimes e_{2 k, 2 k}
\end{gathered}
$$

(iii) for all $k \geq 2$,

$$
\left\|x_{n_{k}}-x_{k}^{\prime}\right\|<\epsilon, \quad\left\|y_{n_{k}}-y_{k}^{\prime}\right\|<\epsilon .
$$

Assuming Lemma 4, let us proceed with the proof of the Theorem:

Suppose that $E$ is stable but $C_{E}$ is not. By Proposition 1 there exist bounded sequences $\left\{x_{n}\right\}_{n=1}^{\infty}$ and $\left\{y_{n}\right\}_{n=1}^{\infty}$ in $C_{E}$ so that, for some $\theta>0$,

$$
\inf _{n>m}\left\|x_{n}+y_{m}\right\| \geq \theta+\sup _{n<m}\left\|x_{n}+y_{m}\right\| \text {. }
$$

Notice that if $\left\{n_{k}\right\}_{k=1}^{\infty}$ is any increasing sequence of positive integers then

$$
\inf _{k>l}\left\|x_{n_{k}}+y_{n_{l}}\right\| \geq \theta+\sup _{k<l}\left\|x_{n_{k}}+y_{n_{l}}\right\| \text {. }
$$

Using Corollary 3 and Lemma 4 with $\epsilon=\theta / 5$ we can assume by (7) that the sequences $\left\{x_{k}^{\prime}\right\}_{k=2}^{\infty}$ and $\left\{y_{k}^{\prime}\right\}_{k=2}^{\infty}$ given by (3) and (4) respectively satisfy

$$
\inf _{n>m \geq 2}\left\|x_{n}^{\prime}+y_{m}^{\prime}\right\| \geq \frac{\theta}{5}+\sup _{2<n<m}\left\|x_{n}^{\prime}+y_{n}^{\prime}\right\| .
$$

Let

$$
\begin{aligned}
a= & x_{2}^{\prime}-z_{2} \otimes e_{4,4}+y_{3}^{\prime}-w_{3} \otimes e_{6,6} \\
= & x_{1,1} \otimes e_{1,1}+x_{1,2} \otimes e_{1,3}+x_{2,1} \otimes e_{3,1}+x_{2,2} \otimes e_{3,3} \\
& +y_{1,1} \otimes e_{1,1}+y_{1,2} \otimes e_{1,5}+y_{2,1} \otimes e_{5,1}+y_{2,2} \otimes e_{5,5}
\end{aligned}
$$


and define, for $k \geq 2$,

$$
a_{k}=a+z_{k} \otimes e_{2 k+4,2 k+4}, \quad b_{k}=w_{k} \otimes e_{2 k+3,2 k+3} .
$$

Clearly, for all $n \neq m$ we have $\left\|x_{n}^{\prime}+y_{m}^{\prime}\right\|=\left\|a_{n}+b_{m}\right\|$. Let $s\left(a_{n}\right)=\left(s_{i}\left(a_{n}\right)\right)_{i=1}^{\infty}$ and $s\left(b_{m}\right)=\left(s_{i}\left(b_{m}\right)\right)_{i=1}^{\infty}$ be the sequences of $s$-numbers of $a_{n}$ and $b_{m}$ respectively, then since $a_{n}$ and $b_{m}$ are two-sided disjointly supported, i.e. they have orthogonal ranges and orthogonal cokernels, we obtain $\left\|a_{n}+b_{m}\right\|_{C_{E}}=\left\|s\left(a_{n}\right)+s\left(b_{m}\right)\right\|_{E}$. So, the bounded sequences $\left\{s\left(a_{k}\right)\right\}_{k=2}^{\infty}$ and $\left\{s\left(b_{k}\right)\right\}_{k=2}^{\infty}$ in $E$ satisfy

$$
\inf _{n>m}\left\|s\left(a_{n}\right)+s\left(b_{m}\right)\right\|_{E} \geq \frac{\theta}{5}+\sup _{n<m}\left\|s\left(a_{n}\right)+s\left(b_{m}\right)\right\|_{E}
$$

which contradicts the fact that $E$ is stable.

PROOF OF LEMMA 4. We present the proof in the case of one sequence (or, equivalently, $x_{n}=y_{n}$ for all $n$ ). The general case follows from this special case by applying the same arguments for the two series simultaneously (see the remark after the formulation of Lemma 6).

Observe first, the following:

PROPOSITION 5. Let $X$ be a Banach space having a boundedly complete Schauder finite-dimensional decomposition $\left\{X_{m}\right\}_{m=1}^{\infty}$, and let $\left\{x_{n}\right\}_{n=1}^{\infty}$ be any bounded sequence in $X$. Then for every $\epsilon>0$ there exist two increasing sequences $\left\{n_{k}\right\}_{k=1}^{\infty}$ and $\left\{m_{k}\right\}_{k=1}^{\infty}$ of positive integers, and elements $x \in \operatorname{span}\left\{X_{m}\right\}_{m=1}^{m_{1}}$ and $z_{k} \in$ $\operatorname{span}\left\{X_{m}\right\}_{m=m_{k}+1}^{m_{k+1}}, k=1,2, \ldots$, so that

$$
\left\|x_{n_{k}}-\left(x+z_{k}\right)\right\|<\epsilon
$$

for all $k \geq 2$.

The routine proof is omitted.

It follows that it is enough to consider in the proof of Lemma 4 bounded sequences $\left\{x_{n}\right\}_{n=1}^{\infty}$ in $C_{E}$ having the form $x_{n}=x+z_{n}$, where for some increasing sequence $\left\{m_{n}\right\}_{n=1}^{\infty}$ of positive integers the following hold:

$$
\begin{cases}x(i, j)=0 & \text { if } \max \{i, j\}>m_{1} \\ z_{n}(i, j)=0 & \text { if } \max \{i, j\}>m_{n+1} \text { or } \max \{i, j\} \leq m_{n}\end{cases}
$$

Next, since the case $\liminf \inf _{n \rightarrow \infty}\left\|z_{n}\right\|=0$ is trivial, we can assume that $\left\|z_{n}\right\|=1$ without loss of generality, i.e., $\left\{z_{n}\right\}_{n=1}^{\infty}$ is a normalized shell-block basic sequence. Let us now recall the following simplified versions of [1, Corollary 2.8] (see also [1, Theorem 2.4]).

LEMMA 6. (a) Let $\left\{z_{n}\right\}_{n=1}^{\infty}$ be a normalized shell-block basic sequence in $C_{E}$, and assume that $c_{0} \nsubseteq C_{E}$. Then for every $\epsilon>0$ there exist a subsequence $\left\{z_{n_{k}}\right\}_{k=2}^{\infty}$, a sequence $\left\{z_{k}^{\prime}\right\}_{k=2}^{\infty}$ in $C_{E}$ and two unitary operators $u$ and $v$ so that, for every $k \geq 2$,

$$
u z_{k}^{\prime} v=a \otimes e_{k, 1}+b \otimes e_{1, k}+c_{k} \otimes e_{k, k}
$$

for some finite matrices $a, b$ and $\left\{c_{k}\right\}_{k=2}^{\infty}$, and so that

$$
\left\|z_{n_{k}}-z_{k}^{\prime}\right\|<\epsilon, \quad k=2,3, \ldots
$$

(b) Assume, moreover, that $\left\{z_{n}\right\}_{n=1}^{\infty}$ are two-sided disjointly supported. Then for every $m \in \mathbf{N}$ the $\left\{z_{k}^{\prime}\right\}_{k=2}^{\infty}$ and $u, v$ can be constructed so that

$$
u z_{k}^{\prime} v=\left(a_{1,1} \otimes e_{1,1}+a_{1,2} \otimes e_{1,2}+a_{2,1} \otimes e_{2,1}+z_{k}^{\prime \prime} \otimes e_{2,2}\right) \otimes e_{k, k}
$$

and (11) hold for every $k \geq 2$, and so that $a_{1,1}, a_{1,2}$ and $a_{2,1}$ are $m \times m$ matrices, and $z_{k}^{\prime \prime}$ are finite (not necessarily $m \times m$ ) matrices. 
We remark that, by the proof of Theorem 2.4 of [1], Lemma 6 applies to two sequences simultaneously (i.e., one pair of unitary operators $u, v$ works for the two sequences). Also, it will be more convenient for us to identify the approximating elements $\left\{z_{k}^{\prime}\right\}$ with the elements $\left\{u z_{k}^{\prime} v\right\}$ which have the "nice" structure (10) or (12), and so we shall simply assume that the $\left\{z_{k}^{\prime}\right\}$ are equal to the $\left\{u z_{k}^{\prime} v\right\}$. This convention will simplify the notation a great deal.

By applying Lemma $6(\mathrm{a})$ to the sequence $\left\{z_{n}\right\}_{n=1}^{\infty}$ of (9), we get a subsequence $\left\{x_{n}\right\}_{n \in N_{1}}$ of $\left\{x_{n}\right\}_{n=1}^{\infty}$, a positive integer $m \geq m_{1}, m \times m$ matrices $a_{1}, b_{1}$, and $c_{1}$ and a sequence $\left\{z_{n}^{(1)}\right\}_{n \in N_{1}}$ in $C_{E}\left(\equiv C_{E}\left(l_{2} \otimes l_{2}\right)\right)$ so that if

$$
\tilde{w}_{n}^{(1)}=a_{1} \otimes e_{1,1}+b_{1} \otimes e_{1, n}+c_{1} \otimes e_{n, 1}, \quad n \in N_{1},
$$

then

$$
\left\|x_{n}-\left(\tilde{w}_{n}^{(1)}+z_{n}^{(1)} \otimes e_{n, n}\right)\right\| \leq \epsilon / 4, \quad n \in N_{1} .
$$

Next, by applying Lemma 6 (b) to the sequence $\left\{z_{n}^{(1)} \otimes e_{n, n}\right\}_{n \in N_{1}}$, we get a subsequence $\left\{x_{n}\right\}_{n \in N_{2}}$ of $\left\{x_{n}\right\}_{n \in N_{1}}$, and sequences $\left\{w_{n}^{(2)}\right\}_{n \in N_{2}}$ and $\left\{z_{n}^{(2)}\right\}_{n \in N_{2}}$ in $C_{E}\left(\equiv C_{E}\left(l_{2} \otimes l_{2} \otimes l_{2}\right)\right)$, so that

$$
w_{n}^{(2)}=\left(a_{2} \otimes e_{1,1}+b_{2} \otimes e_{1,2}+c_{2} \otimes e_{2,1}\right) \otimes e_{n, n}, \quad n \in N_{2},
$$

where $a_{2}, b_{2}$ and $c_{2}$ are $m \times m$ matrices, and so that if we represent the $\tilde{w}_{n}^{(1)}$ as elements of $C_{E}\left(l_{2} \otimes l_{2} \otimes l_{2}\right)$ by

$$
w_{n}^{(1)}=a_{1} \otimes e_{1,1} \otimes e_{1,1}+b_{1} \otimes e_{1,1} \otimes e_{1, n}+c_{1} \otimes e_{1,1} \otimes e_{n, 1}, \quad n \in N_{2},
$$

then

$$
\left\|x_{n}-\left(w_{n}^{(1)}+w_{n}^{(2)}+z_{n}^{(2)} \otimes e_{2,2} \otimes e_{n, n}\right)\right\| \leq \epsilon\left(1 / 4+1 / 4^{2}\right)
$$

for all $n \in N_{2}$. Let $n_{2}$ be the first element in $N_{2}$, and continue the construction by applying Lemma $6(\mathrm{~b})$ to the sequence $\left\{z_{n}^{(2)} \otimes e_{2,2} \otimes e_{n, n}\right\}_{n \in N_{2} \backslash\left\{n_{2}\right\}}$.

By an obvious inductive process, using Lemma 6(b) in each step, we construct a decreasing sequence $\left\{N_{k}\right\}_{k=1}^{\infty}$ of infinite subsets of $\mathbf{N}$, so that if $n_{k}$ is the first element of $N_{k}$ then $N_{k+1} \subseteq N_{k} \backslash\left\{n_{k}\right\}, k \geq 2$, and sequences $\left\{w_{n}^{(k)}\right\}_{n \in N_{k}},\left\{z_{n}^{(k)}\right\}_{n \in N_{k}}$ so that, for $k \geq 2$ and $n \in N_{k}$,

$$
w_{n}^{(k)}=\left(a_{k} \otimes e_{k-1, k-1}+b_{k} \otimes e_{k-1, k}+c_{k} \otimes e_{k, k-1}\right) \otimes e_{n, n}
$$

where $a_{k}, b_{k}$ and $c_{k}$ are $m \times m$ matrices, and so that, for all $n \in N_{k}$,

$$
\left\|x_{n}-\left(\sum_{j=1}^{k} w_{n}^{(j)}+z_{n}^{(k)} \otimes e_{k, k} \otimes e_{n, n}\right)\right\| \leq \epsilon \cdot \sum_{j=1}^{k} 4^{-j} .
$$

Consider now the infinite sequence $\left\{u_{k}\right\}_{k=2}^{\infty}$ defined by

$$
u_{k}=a_{k} \otimes e_{k-1, k-1}+b_{k} \otimes e_{k-1, k}+c_{k} \otimes e_{k, k-1} \text {. }
$$

Clearly,

and

$$
\sup _{k \geq 2}\left\|\sum_{\substack{j=2 \\ j \text { even }}}^{k} u_{j}\right\|=\sup _{k \geq 2}\left\|\sum_{\substack{j=2 \\ j \text { even }}}^{k} w_{n_{k}}^{(j)}\right\|<\infty
$$

$$
\sup _{k \geq 2}\left\|\sum_{\substack{j=2 \\ j \text { odd }}}^{k} u_{j}\right\|=\sup _{k \geq 2}\left\|\sum_{\substack{j=2 \\ j \text { odd }}}^{k} w_{n_{k}}^{(j)}\right\|<\infty .
$$


Since the shell decomposition in $C_{E}$ is boundedly complete this easily implies that $\sum_{k=2}^{\infty} u_{k}$ converges in $C_{E}$. In particular, there exists a $k_{0} \geq 2$ so that for all $k \geq k_{0}+1$

$$
\left\|\sum_{j=k_{0}+1}^{k} w_{n_{k}}^{(j)}\right\|=\left\|\sum_{j=k_{0}+1}^{k} u_{j}\right\| \leq \epsilon / 4 .
$$

For $k>k_{0}$ let

$$
x_{k}^{\prime}=\sum_{j=1}^{k_{0}} w_{n_{k}}^{(j)}+z_{n_{k}}^{(k)} \otimes e_{k, k} \otimes e_{n_{k}, n_{k}} .
$$

Now, for $k>k_{0}$

$$
\begin{aligned}
\left\|x_{n_{k}}-x_{k}^{\prime}\right\| & \leq\left\|x_{n_{k}}-\left(\sum_{j=1}^{k} w_{n_{k}}^{(j)}+z_{n_{k}}^{(k)} \otimes e_{k, k} \otimes e_{n_{k}, n_{k}}\right)\right\|+\left\|\sum_{j=k_{0}+1}^{k} w_{n_{k}}^{(j)}\right\| \\
& \leq \epsilon\left(\sum_{j=1}^{k} 4^{-j}+1 / 4\right)<\epsilon .
\end{aligned}
$$

Observing that $\sum_{j=1}^{k_{0}} w_{n_{k}}^{(j)}$ and $z_{n_{k}}^{(k)} \otimes e_{k, k} \otimes e_{n_{k}, n_{k}}$ are two-sided disjointly supported we get by reblocking and by passing to a new tensor product representation (of $C_{E}$ and $\left.C_{E}\left(l_{2} \otimes l_{2}\right)\right)$ :

$$
\begin{aligned}
x_{k}^{\prime}= & x_{1,1} \otimes e_{1,1}+x_{1,2} \otimes e_{1,2 k-1}+x_{2,1} \otimes e_{2 k-1,1} \\
& +x_{2,2} \otimes e_{2 k-1,2 k-1}+\tilde{z}_{k} \otimes e_{2 k, 2 k}, \quad\left(k>k_{0}\right) .
\end{aligned}
$$

This clearly completes the proof of Lemma 4 in the case of one sequence. As we remarked during the proof, the case of two sequences follows from the case of one sequence since Lemma 6 holds for two sequences simultaneously.

ACKNOWLEDGEMENT. This paper was written while the author was visiting Odense University. He is happy to express his thanks to his colleagues for arranging his visit and for the warm hospitality in Odense.

\section{REFERENCES}

1. J. Arazy, Basic sequences, embeddings, and the uniqueness of the symmetric structure in unitary matrix spaces, J. Funct. Anal. 40 (1981), 301-340.

2. J. L. Krivine and B. Maurey, Espaces de Banach stables, Israel J. Math. 39 (1981), 273-295.

3. S. Guerre and J. T. Lapresté, Quelques propriétés des espaces de Banach stables, Israel J. Math. 39 (1981), 247-254.

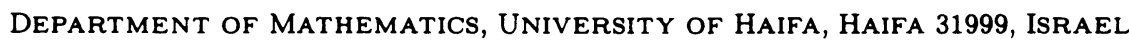

